\title{
The effect of initial intravitreal tissue plasminogen activator and gas injection on vision improvement in patients with submacular haemorrhage associated with age-related macular degeneration
}

\author{
Seung Ha Lee ${ }^{1}$ Sang Joon Lee $\mathbb{1}^{1} \cdot$ Yong II Shin ${ }^{2} \cdot$ Hyung Bin $\operatorname{Lim}^{3} \cdot$ Jung Yeul Kim $\mathbb{I}^{3} \cdot$ Yong Seop Han ${ }^{4}$. \\ Ki Yup Nam $\mathbb{1}^{5}$
}

Received: 21 July 2020 / Revised: 30 November 2020 / Accepted: 18 December 2020 / Published online: 9 January 2021

(c) The Author(s), under exclusive licence to The Royal College of Ophthalmologists 2021

\begin{abstract}
Purpose To compare visual improvements between initial intravitreal t-PA with gas injection before anti-vascular endothelial growth factor (VEGF) and anti-VEGF injection monotherapy for submacular haemorrhage (SMH) associated with age-related macular degeneration (AMD).

Methods We retrospectively reviewed medical records of naive patients treated with intravitreal t-PA with gas injection before anti-VEGF (Group 1) or only with intravitreal anti-VEGF injection (Group 2) for SMH [disc area (DA) $\geq 2$ ] associated with AMD from two institutions. Both groups received 3 monthly loads of anti-VEGF injections followed by injections as needed for AMD treatment. Changes in best-corrected visual acuity (BCVA, logMAR) between the initial visit and after 6 months of treatment were compared between two groups.

Results A total of 82 patients were enroled. Of these, 32 patients and 50 patients were grouped in Groups 1 and 2, respectively. The mean change in BCVA over 6 months for Group 1 was $-0.52 \pm 0.88$, which was significantly larger $(p=$ $0.044)$ than the mean change for Group $2(-0.15 \pm 0.58)$. We compared visual improvements between the two groups based on the following SMH size categories: $\leq 5,>5$, and $\leq 15$, and $>15$ DA. When the SMH size was $\leq 5$, or $>5$ and $\leq 15$ DA, the mean change in BCVA was larger for Group 1 than for Group 2, but this difference was not significant. When SMH size was $>15$ DA, Group 1 patients exhibited a mean visual improvement of $-0.79 \pm 0.80$, which was significantly greater $(p=$ $0.029)$ than that of Group $2(-0.06 \pm 0.67)$.

Conclusions Patients that were primarily treated for SMH associated with AMD using t-PA and gas injection (followed by anti-VEGF injection) exhibited better visual improvement than those treated with anti-VEGF monotherapy, especially in patients exhibiting larger SMH sizes (>15 DA) at the initial visit.
\end{abstract}

Ki Yup Nam

oksnam1231@daum.net

1 Department of Ophthalmology, College of Medicine, Kosin University, Busan, South Korea

2 Rhee's Eye Hospital, Daejeon, South Korea

3 Department of Ophthalmology, College of Medicine, Chungnam National University, Daejeon, South Korea

4 Department of Ophthalmology, College of Medicine, Gyeongsang National University, Jinju, South Korea

5 Department of Ophthalmology, Chungnam National University Sejong Hospital, Sejong, South Korea

\section{Introduction}

Submacular haemorrhage (SMH) is an uncommon complication of age-related macular degeneration (AMD) with a poor visual prognosis $[1,2]$. The poor prognosis is associated with the toxicity of the SMH to the retina. SMHs can create a barrier preventing nutrients and metabolic products from moving between the retina and retinal pigment epithelium, and iron from the blood can be toxic to the retina $[3,4]$.

The treatments for SMH can be largely divided into intravitreal injections and surgical treatments. Anti-vascular endothelial growth factor (VEGF) and gas with or without tissue plasminogen activators (t-PAs) can be used by the intravitreal injection. The surgical treatment is the vitrectomy with multiple procedures such as the removal of 
choroidal neovascularization lesions, macular translocation, subretinal t-PA injection, and SMH drainage [5].

Intravitreal injections are less invasive than vitrectomy and can be used in out-patient clinics. Several methods of intravitreal injection have been reported to show good anatomical and functional results without surgery [6-12].

Some doctors use intravitreal anti-VEGF injection only because it is much easier to perform compared to gas injection. In addition, there may be concerns about complications after gas injection, including vitreous haemorrhage, increase of intraocular pressure, retinal pigment epithelial tearing, and retinal detachment [7, 8, 13-17]. Also, the prognosis of SMH patients is believed to be poor regardless of treatment. However, an intravitreal gas injection with or without additional t-PA injection can reduce the duration that the retina contacts subretinal clots, and early removal of the SMH can reveal underlying diseases for further investigation [5]. Each of these less invasive procedures has its own advantages and disadvantages; hence, there is no standard treatment option for SMH in AMD patients. In addition, there were few studies have analysed the effect of initial pneumatic displacement with t-PA before anti-VEGF injections compared to intravitreal antiVEGF injection monotherapy.

Thus, we investigated visual improvements after intravitreal t-PA with gas injection (followed by anti-VEGF injections) and anti-VEGF monotherapy for SMH associated with AMD.

\section{Methods}

We retrospectively reviewed the medical records of naive AMD patients with SMH who were treated with intravitreal t-PA, gas injections, and intravitreal anti-VEGF injections at two institutions. The patients at Kosin University Hospital (Busan, South Korea) received intravitreal t-PA and gas injections as initial treatments for SMH and those at Chungnam National University Hospital (Daejeon, South Korea) only received anti-VEGF injections. Our study protocol was approved by the Institutional Review Board of Kosin University Hospital (Kosin IRB No. 2019-05-025001) and this study adhered to the tenets of the Declaration of Helsinki.

Naive AMD patients with SMH sizes $\geq 2$ disc area (DA) and with ages $>50$ years were enroled in the study. Patients with diabetic retinopathy, retina vessel occlusion, glaucoma, uveitis, or trauma history, which might affect visual acuity, were excluded. Cases with poor fundus visibility due to anterior segment problems such as cornea opacity or severe cataract were also excluded. In addition, patients were excluded if their follow-up period was less than 6 months or if they had previous intraocular surgery other than cataract surgery. Cases involving visual acuity affected by complications of SMH and treatments for rhegmatogenous retinal detachment (RRD) during the follow-up period were also excluded.

The patients were diagnosed with AMD using fluorescein angiography (FA) and indocyanine green angiography (ICGA; HRA Spectralis; Heidelberg Engineering, Heidelberg, Germany). If the SMH was too thick to define the choroidal vascular abnormalities, FA/ICGA was performed after reduction of the SMH.

From the medical records, data on age, sex, past medical history (diabetes mellitus, hypertension, or anticoagulant use), duration of symptoms, lens status, best-corrected visual acuity (BCVA) at the initial visit and at 6 months of follow-up, and the type of AMD were extracted. The SMH size at the initial visit was measured based on the SMH size using fundus photography.

If the patient was taking anticoagulants, they were told to stop for a week when given consent before treatment. Patients with cardiovascular disease (stroke, ischaemic heart disease, etc.) were referred to the relevant department.

\section{Procedure}

At Chungnam National University Hospital, intravitreal anti-VEGF injections were performed as an initial treatment. After mydriasis (Mydrin- $\mathrm{P}^{\circ}$; Santen Pharmaceutical, Osaka, Japan) and topical anaesthesia $(0.5 \%$ Alcaine Alcon, Fort Worth, TX, USA), half-potadine (5\%) was instilled on the conjunctival sac. After draping of the eyelid, anti-VEGF was injected at $3.75 \mathrm{~mm}$ from the limbus in phakic patients and at $3.25 \mathrm{~mm}$ in pseudophakic patients. After injection, antibiotic eye drops were used for 1 week. After 3 monthly loading injections, injections were carried out as needed when subretinal fluid or pigment epithelial detachment recurred or worsened. These patients were grouped in the intravitreal anti-VEGF monotherapy group (Group 2).

At Kosin University Hospital, after preparation similar to that at Chungnam National University Hospital, $50 \mu \mathrm{g} / 0.05$ $\mathrm{mL}$ t-PA (Actilyse ${ }^{\bullet}$, Boehringer Ingelheim $\mathrm{GmbH}$, Ingelheim am Rhein, Germany) was injected intravitreally in the same way as the first injection. After $5 \mathrm{~min}$, intravitreal gas injection $\left(0.3 \mathrm{~mL}\right.$ of $100 \% \mathrm{C}_{3} \mathrm{~F}_{8}$ gas; Matheson Tri-Gas, Montgomeryville, PA, USA) was performed, followed by anterior chamber paracentesis to reduce the intraocular pressure. The patients were then requested to assume a prone position (facing downward) for 1-2 weeks, based on the status of the SMH. One week later, anti-VEGF was injected in a similar manner as at Chungnam National University Hospital (three loading injections followed by as-needed injections). These patients were grouped in Group 1. 
Each physician selected between bevacizumab, ranibizumab, and aflibercept as the anti-VEGF. Vitrectomy was performed if breakthrough vitreous haemorrhage occurred and combined cataract surgery was also performed in phakic patients.

The main outcome was the BCVA in each group at the initial visit and at 6 months after initial treatments, and changes in BCVA between the initial visit and at 6 months were compared between groups.

SPSS software for Windows (ver. 20.0; SPSS, Chicago, IL, USA) was used for statistical analyses. For comparisons of the mean values of continuous variables, an independent $t$-test was performed. The chi-square test was used for discontinuous variables, and the Mann-Whitney $U$ test was used for nonparametric comparisons of mean values.

\section{Results}

\section{Baseline characteristics}

A total of 82 patients were enroled-32 patients (Group 1) were initially treated with initial intravitreal injections of t-PA and gas before anti-VEGF injections and 50 patients (Group 2 ) were treated only with anti-VEGF injections. The baseline characteristics of the patients are shown in Table 1. There were no significant differences between the two groups except for BCVA at the initial visit. The mean initial logarithm of the minimum angle of resolution (logMAR) BCVA was $1.29 \pm$ 0.63 for Group 1 and $1.00 \pm 0.59$ for Group 2, and these values were significantly different $(p=0.040)$.

\section{Anti-VEGF treatments for SMH}

All patients were treated using one of the anti-VEGF agents (bevacizumab, ranibizumab, or aflibercept). A total of 14 (43.8\%), 8 (25.0\%), and 10 (31.2\%) patients in Group 1, and $21(42.0 \%), 19(36.0 \%)$, and $10(20.0 \%)$ patients in Group 2 were treated with bevacizumab, ranibizumab, or aflibercept, respectively. The mean numbers of bevacizumab, ranibizumab, and aflibercept injections were $4.00 \pm 0.7,3.6 \pm 0.6$, and $3.5 \pm 0.5$ for Group 1 , and $3.4 \pm$ $0.8,3.5 \pm 0.7$, and $3.1 \pm 0.3$ for Group 2, respectively, and did not differ significantly between groups $(p=0.114$, 0.587 , and 0.173 , respectively).

\section{Changes in BCVA after treatments}

The mean $\log$ MAR BCVA at 6 months after initial treatment did not differ significantly ( $p=0.683$ ) between Group $1(0.76 \pm 0.83)$ and Group $2(0.84 \pm 0.79)$. However, the mean change in BCVA between the initial visit and at 6 months for Group 1 was $-0.52 \pm 0.88$, which was
Table 1 Baseline characteristics in intravitreal tissue plasminogen activator and gas injection group (Group 1) and anti-vascular endothelial growth factor injection group (Group 2) as an initial treatment.

\begin{tabular}{|c|c|c|c|}
\hline & $\begin{array}{l}\text { Group 1 } \\
(n=32)\end{array}$ & $\begin{array}{l}\text { Group 2 } \\
(n=50)\end{array}$ & $p$ value \\
\hline Age & $68.2( \pm 11.0)$ & $72.9( \pm 10.4)$ & $0.102^{\mathrm{i}}$ \\
\hline $\operatorname{Sex}(n, \%)$ & & & $0.846^{\mathrm{j}}$ \\
\hline Male & $17(56.2 \%)$ & $30(60.0 \%)$ & \\
\hline Female & $14(43.8 \%)$ & $20(40.0 \%)$ & \\
\hline $\mathrm{DM}^{\mathrm{a}}(n, \%)$ & $10(31.3 \%)$ & $10(20.0 \%)$ & $0.274^{\mathrm{j}}$ \\
\hline $\operatorname{HTN}^{\mathrm{b}}(n, \%)$ & $15(46.9 \%)$ & $27(54.0 \%)$ & $0.695^{\mathrm{j}}$ \\
\hline Anticoagulant $(n, \%)$ & $7(21.9 \%)$ & $15(30.0 \%)$ & $0.710^{\mathrm{j}}$ \\
\hline Aspirin & $4(12.5 \%)$ & $11(22.0 \%)$ & \\
\hline Clopidogrel & $1(3.1 \%)$ & $2(4.0 \%)$ & \\
\hline Warfarin & $2(6.3 \%)$ & $2(4.0 \%)$ & \\
\hline Symptom duration (days) & $13.5( \pm 12.1)$ & $12.2( \pm 10.3)$ & $0.736^{\mathrm{i}}$ \\
\hline Lens status $(n, \%)$ & & & $0.497^{\mathrm{j}}$ \\
\hline Phakic & $24(75.0 \%)$ & $36(72.0 \%)$ & \\
\hline Pseudophakic & $8(25.0 \%)$ & $14(28.0 \%)$ & \\
\hline $\begin{array}{l}\mathrm{BCVA}^{\mathrm{c}} \text { at initial visit } \\
(\log \mathrm{MAR})\end{array}$ & $1.29( \pm 0.63)$ & $1.00( \pm 0.59)$ & $0.040^{\mathrm{i} *}$ \\
\hline $\begin{array}{l}\mathrm{SMH}^{\mathrm{d}} \text { size at initial visit } \\
\text { (disc area) }\end{array}$ & $15.4( \pm 14.1)$ & $14.2( \pm 12.4)$ & $0.683^{\mathrm{i}}$ \\
\hline Type of $\operatorname{AMD}^{\mathrm{e}}(n, \%)$ & & & $0.206^{\mathrm{j}}$ \\
\hline $\mathrm{CNV}^{\mathrm{f}}$ & $9(28.1 \%)$ & $23(46.0 \%)$ & \\
\hline $\mathrm{PCV}^{\mathrm{g}}$ & $23(71.9 \%)$ & $25(50.0 \%)$ & \\
\hline $\mathrm{RAP}^{\mathrm{h}}$ & 0 & $2(4.0 \%)$ & \\
\hline
\end{tabular}

${ }^{\mathrm{a}}$ Diabetes mellitus.

${ }^{\mathrm{b}}$ Hypertension.

${ }^{\mathrm{c}}$ Best-corrected visual acuity.

${ }^{\mathrm{d}}$ Submacular haemorrhage.

${ }^{\mathrm{e}}$ Age-related macular degeneration.

${ }^{\mathrm{f}}$ Choroidal neovascularization.

${ }^{g}$ Polypoidal choroidal vasculopathy.

${ }^{\mathrm{h}}$ Retinal angiomatous proliferation.

${ }^{\mathrm{i}}$ Independent $t$-test.

${ }^{\mathrm{j}}$ Chi-square test.

$* p<0.050$.

significantly greater $(p=0.044)$ than that for Group 2 $(-0.15 \pm 0.58)$

We tried to analysis the effect of both treatments according to the SMH size and compared visual improvements between the two groups based on SMH size: $\leq 5,>5$, and $\leq 15$, and $>15$ DA. When the SMH sizes were $\leq 5 \mathrm{DA}$ $(n=8$, Group $1 ; n=17$, Group 2$)$ or $>5$ and $\leq 15$ DA $(n=$ 15 , Group $1 ; n=16$, Group 2), the mean changes in BCVA were $-0.57 \pm 0.40$ or $-0.33 \pm 1.09$ for Group 1 , respectively, and $-0.22 \pm 0.42$ or $-0.18 \pm 0.67$ for Group 2, respectively. These values did not differ significantly between the two groups ( $p=0.157$ or 0.572 , respectively). 
When the SMH sizes were $>15$ DA $(n=9$, Group $1 ; n=$ 17 , Group 2), the mean visual improvement in Group 1 was $-0.79 \pm 0.80$, which was significantly greater $(p=0.029)$ than that of Group $2(-0.06 \pm 0.67$; Fig. 1). These results were associated with early displacement of SMH from the posterior pole of retina (Fig. 2).

\section{Treatment complications}

Vitreous haemorrhage occurred in 10 (31.2\%) and 17 (34.0\%) patients in Groups 1 and 2, respectively, but proportions did not differ significantly between groups $(p=0.844)$. Vitrectomy was necessary for six $(18.7 \%)$ patients in Group 1 and five $(8.0 \%)$ patients in Group $2(p=0.137)$. Re-bleeding did not occur for 6 months in both groups. There were one and

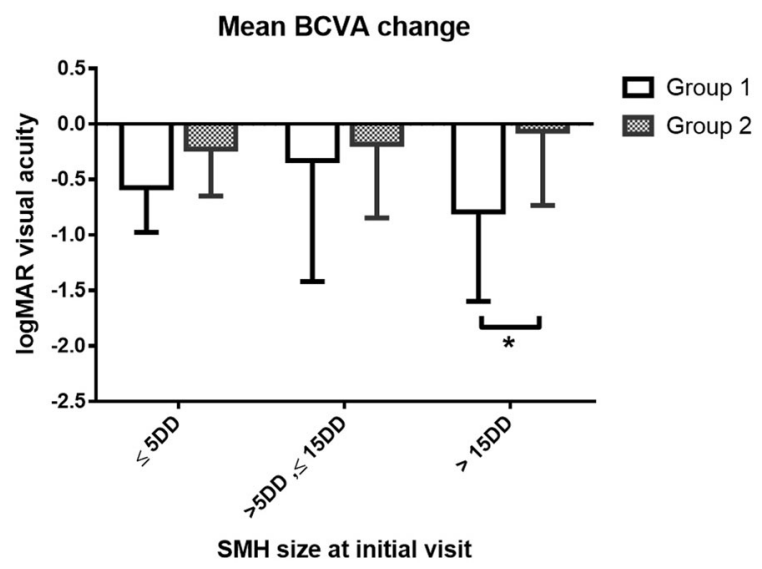

Fig. 1 Comparison of mean changes in BCVA from the baseline between two treatment groups based on SMH size as follows: $\leq \mathbf{5}$, $>\mathbf{5}$, and $\leq \mathbf{1 5}$, and $>\mathbf{1 5} \mathrm{DA}$. Group 1 patients received t-PA and gas injections (followed by anti-VGF), whereas Group 2 patients only received anti-VEGF injections. When the SMH sizes were $>15$ DA, the mean visual improvement observed in Group 1 was significantly greater than that of Group $2(p=0.029)$. two cases of RPE tear involving fovea in Groups 1 and 2, respectively ( $p=0.837$ ). One Group 1 patient (t-PA and gas injections followed by anti-VEGF injections) and two Group 2 patients (only anti-VEGF injections) developed RRD and needed vitrectomy with silicone oil injection. These RRD cases were excluded from further analysis.

\section{Discussion}

SMH associated with AMD is rare but has a poor visual prognosis if it remains untreated [4, 18, 19]. SMH can be treated non-surgically (e.g., intravitreal injections) or surgically (e.g., pars plana vitrectomy). Non-surgical treatments, which include intravitreal gas injections with or without t-PA injections plus intravitreal anti-VEGF injections, and anti-VEGF monotherapy, have led to favourable improvement of visual acuity [6, 8, 11, 12, 19-25]. These therapies, which are less invasive than vitrectomy, are widely used in clinical practice. However, there are no standard guidelines for treatment decisions; physicians select treatment modalities according to their preferences.

We assumed that the pneumatic displacement of SMH may be helpful for visual improvement after treatment compared to anti-VEGF monotherapy because the former reduces retinal damage by limiting retinal exposure time to the toxic or barrier effects of the haemorrhage [3, 4]. Although we could not analyse the change of macular thickness or SMH thickness due to difference of OCT machines in two institutions, t-PA with gas injection treatment generally showed early displacement of SMH from the posterior pole of retina compared to anti-VEGF monotherapy group (Fig. 2). Thus, we directly compared visual improvements between patients that received initial intravitreal injections of t-PA and gas before anti-VEGF injections (Group 1) and those that received anti-VEGF monotherapy (Group 2). The mean

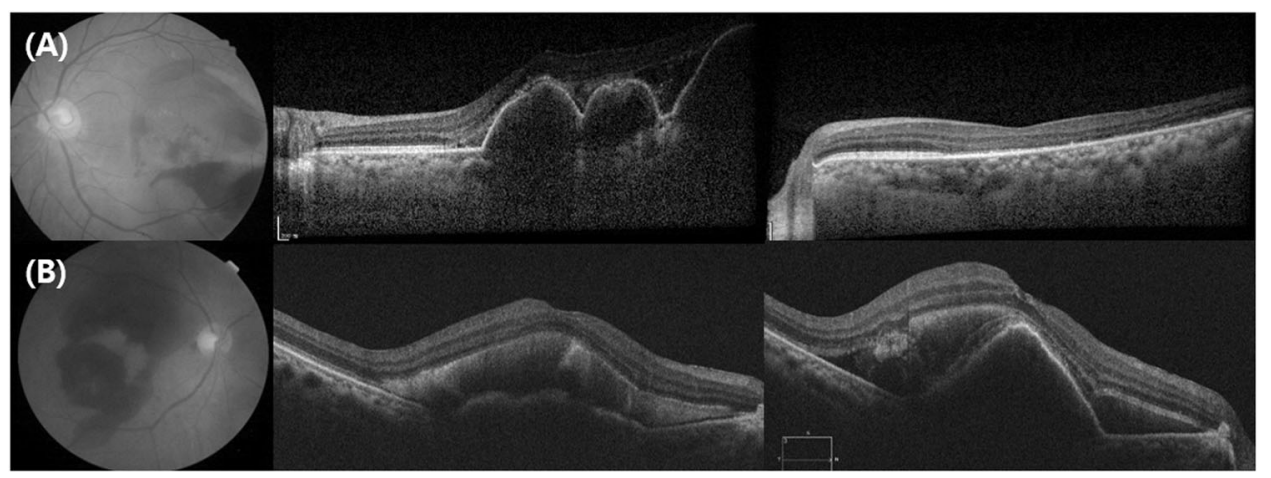

Fig. 2 The representative cases in Groups 1 and 2 with initial SMH size $>15$ disc area. A Optical coherence tomography (OCT) images at Initial visit and 1 week after intravitreal t-PA and gas injection as an initial treatment for submacular haemorrhage (SMH). In a week, SMH successfully migrated to peripheral area from the foveal centre. B OCT at initial visit and 1 month after anti-vascular endothelial growth factor antibody injection. The SMH remained similar for 1 month. 
change in BCVA over 6 months for Group 1 was $-0.52 \pm$ 0.88 , which was significantly larger than the mean change for Group $2(-0.15 \pm 0.58)(p=0.044)$. The early displacement of SMH from posterior pole and reduction of the further retinal damage by t-PA with gas injection as an initial treatment may have resulted in better visual improvement compared to only anti-VEGF injections. Re-bleeding or RPE tear involving fovea during the follow-up period after treatments may affect the final visual outcome. There were no cases with re-bleeding in both groups. RPE tear occurred in Groups 1 and 2, respectively, but the difference was not significant.

There are few reports comparing visual improvements in patients treated with pneumatic displacement with or without t-PA and those treated with anti-VEGF injection monotherapy. Cho et al. [26] reported that pneumatic displacement treatment did not appear to benefit patients with SMH. Changes in visual acuity and central macular thickness (CMT) over 1 year were compared between patients receiving ranibizumab injection monotherapy and gas $\left(\mathrm{C}_{3} \mathrm{~F}_{8}\right.$ or $\mathrm{SF}_{6}$ ) injections with additional ranibizumab, and no significant differences were observed between the two groups. However, the mean SMH sizes at the initial visit were $8.9 \pm 6.6$ DA for patients receiving pneumatic displacement with ranibizumab therapy and 7.5 \pm 5.1 DA for patients receiving ranibizumab monotherapy, which were less than half of those measured in our study $(15.5 \pm 14.1$ DA for Group 1 and 14.2 \pm 12.4 DA for Group 2). In our study, when SMH sizes were large, the visual improvement was significantly greater in Group 1 than in Group 2.

Furthermore, Cho et al. [26] did not use t-PA. By contrast, Sacu et al. [27] reported significant differences in visual improvement between patients receiving gas $\left(\mathrm{SF}_{6}\right)$ with t-PA injection therapy and those receiving bevacizumab monotherapy. Patients in the former group exhibited significant visual improvement from baseline values, whereas those receiving bevacizumab monotherapy did not. However, this study only enroled a very small number of patients, so a direct statistical comparison between the two groups was not possible.

Shin et al. [28] reported that pneumatic displacement with anti-VEGF therapy led to more rapid improvement of central foveal thickness and BCVA within 1 month than anti-VEGF monotherapy, but there were no differences at 6 months. However, when subretinal haemorrhage thickness was more than $450 \mu \mathrm{m}$, the visual outcome at 6 months was better in patients receiving gas with anti-VEGF therapy. We observed similar results in our study. If the SMH size is large, the central portion of the SMH is also thicker. Thus, SMH volume is associated with differences in visual improvement after pneumatic displacement therapy and anti-VEGF monotherapy.

To provide a basis for selecting treatment options according to the initial SMH size, we compared visual improvements between the two groups based on a SMH size classification.
When the SMH sizes were $\leq 5$, or $>5$ and $\leq 15$ DA, the mean changes in BCVA did not differ significantly between the two groups. Only when SMH sizes were $>15$ DA, the visual improvement was significantly greater in Group 1 than in Group 2. Thus, the effect of injections with t-PA and gas as an initial treatment on visual improvement was more pronounced and significant when the initial SMH sizes were $>15$ DA.

There were complications of vitreous haemorrhage after both treatment modalities. The rate of vitreous haemorrhage was similar in both groups and to values reported by previous studies. Although the percentage of severe vitreous haemorrhages needing vitrectomy was higher in Group 1, this difference was not significant between the two groups. One patient treated with injections of t-PA and gas (3.0\%) and two patients treated with only anti-VEGF injections (2.0\%) developed RRD. These rates were comparable to those reported by previous studies. Retinal detachment has been reported as a complication of treatments after both pneumatic displacement and intravitreal anti-VEGF injection $[6,8,22,28,29]$. There were no other significant complications, including endophthalmitis or traumatic lens injury.

This study had some limitations. As a retrospective study involving only two institutions, there were inherent limitations in patient enrolment and grouping. In addition, due to the thick SMH, which was often incorrectly measured, it was not possible to compare OCT parameters such as CMT between the two groups. If we could get the CMT value, it would have helped to explain the difference in vision improvement between the two groups. Also, the number of patients was insufficient to perform subgroup analysis. Therefore, a prospective study with more patients is needed to confirm the results of the present study. Despite its limitations, we consider that this study was meaningful because we can recommend treatment modalities according to initial SMH size, and there are no reports which suggest clear guidelines until now.

In conclusion, injections of t-PA and gas as an initial treatment for SMH associated with AMD led to better visual improvement than anti-VEGF monotherapy, especially in cases with larger SMH sizes at the initial visit. The larger the $\mathrm{SMH}$, the greater the visual improvement by t-PA and gas injections with anti-VEGF injection. Based on the results of the current study, t-PA and gas injections are recommended in cases when SMH sizes are $>15$ DA. Physicians should therefore consider the SMH size at the initial visit when deciding the initial treatment.

\section{Summary}

\section{What was known before}

- Intravitreal injections (anti-VEGF or gas) without vitrectomy were reported as a good treatment option for SMH associated with AMD. Although gas injection may reduce 
damage of inner retinal from blood and lead favourable outcome, some doctors use anti-VEGF only because it is much easier to perform compared to gas injection and there may be concerns about complications after gas injection, such as vitreous haemorrhage and RRD. Also, the prognosis of SMH patients is believed to be poor regardless of treatment. Until now, there were few studies that have analysed the effect of initial pneumatic displacement with tPA before anti-VEGF injections compared to intravitreal anti-VEGF injection monotherapy.

\section{What this study adds}

- In the current study, injections of gas and t-PA as an initial treatment for SMH associated with AMD led to better visual improvement than anti-VEGF monotherapy in general. The larger the SMH, the greater the visual improvement by t-PA and gas injections with antiVEGF injection. Based on the results of the current study, t-PA and gas injections are recommended in cases when SMH sizes are $>15$ DA.

Acknowledgements This work was supported by Chungnam National University Sejong Hospital Research Fund, 2020.

Author contributions Design of study (JYK and KYN); analysis and interpretation of data (SHL, SJL, HBL, YSH, KYN); collection of data (SHL, SJL, YIS, HBL, JYK, KYN); draft of manuscript (SHL and $\mathrm{KYN}$ ) and critical review of article (SJL, YSH, HBL, KYN); and approval of final version of manuscript (SHL and KYN).

\section{Compliance with ethical standards}

Conflict of interest The authors declare that they have no conflict of interest.

Publisher's note Springer Nature remains neutral with regard to jurisdictional claims in published maps and institutional affiliations.

\section{References}

1. Poliner LS, Olk RJ, Burgess D, Gordon ME. Natural history of retinal pigment epithelial detachments in age-related macular degeneration. Ophthalmology. 1986;93:543-51.

2. Scupola A, Coscas G, Soubrane G, Balestrazzi E. Natural history of macular subretinal hemorrhage in age-related macular degeneration. Ophthalmologica. 1999;213:97-102. https://doi.org/10. 1159/000027400.

3. Avery RL, Fekrat S, Hawkins BS, Bressler NM. Natural history of subfoveal subretinal hemorrhage in age-related macular degeneration. Retina. 1996;16:183-9.

4. Hochman MA, Seery CM, Zarbin MA. Pathophysiology and management of subretinal hemorrhage. Surv Ophthalmol. 1997;42:195-213.
5. Stanescu-Segall D, Balta F, Jackson TL. Submacular hemorrhage in neovascular age-related macular degeneration: a synthesis of the literature. Surv Ophthalmol. 2016;61:18-32. https://doi.org/ 10.1016/j.survophthal.2015.04.004.

6. Chen CY, Hooper C, Chiu D, Chamberlain M, Karia N, Heriot WJ. Management of submacular hemorrhage with intravitreal injection of tissue plasminogen activator and expansile gas. Retina. 2007;27:321-8. https://doi.org/10.1097/01.iae. 0000237586.48231.75.

7. Handwerger BA, Blodi BA, Chandra SR, Olsen TW, Stevens TS. Treatment of submacular hemorrhage with low-dose intravitreal tissue plasminogen activator injection and pneumatic displacement. Arch Ophthalmol. 2001;119:28-32.

8. Hattenbach LO, Klais C, Koch FH, Gumbel HO. Intravitreous injection of tissue plasminogen activator and gas in the treatment of submacular hemorrhage under various conditions. Ophthalmology. 2001;108:1485-92.

9. Chang MA, Do DV, Bressler SB, Cassard SD, Gower EW, Bressler NM. Prospective one-year study of ranibizumab for predominantly hemorrhagic choroidal neovascular lesions in agerelated macular degeneration. Retina. 2010;30:1171-6. https://doi. org/10.1097/IAE.0b013e3181dd6d8a.

10. Iacono P, Parodi MB, Introini U, La Spina C, Varano M, Bandello F. Intravitreal ranibizumab for choroidal neovascularization with large submacular hemorrhage in age-related macular degeneration. Retina. 2014;34:281-7. https://doi.org/10.1097/ IAE.0b013e3182979e33.

11. Shienbaum G, Garcia Filho CA, Flynn HW Jr, Nunes RP, Smiddy WE, Rosenfeld PJ. Management of submacular hemorrhage secondary to neovascular age-related macular degeneration with antivascular endothelial growth factor monotherapy. Am J Ophthalmol. 2013;155:1009-13. https://doi.org/10.1016/j.ajo.2013.01. 012 .

12. Stifter E, Michels S, Prager F, Georgopoulos M, Polak K, Hirn C, et al. Intravitreal bevacizumab therapy for neovascular age-related macular degeneration with large submacular hemorrhage. Am J Ophthalmol. 2007;144:886-92. https://doi.org/10.1016/j.ajo.2007. 07.034.

13. Hassan AS, Johnson MW, Schneiderman TE, Regillo CD, Tornambe PE, Poliner LS, et al. Management of submacular hemorrhage with intravitreous tissue plasminogen activator injection and pneumatic displacement. Ophthalmology. 1999;106:1900-6. https://doi.org/10.1016/S0161-6420(99) 90399-8.

14. Johnson MW. Pneumatic displacement of submacular hemorrhage. Curr Opin Ophthalmol. 2000;11:201-6.

15. Kokame GT. Vitreous hemorrhage after intravitreal tissue plasminogen activator (t-PA) and pneumatic displacement of submacular hemorrhage. Am J Ophthalmol. 2000;129:546-7.

16. Wu TT, Kung YH, Hong MC. Vitreous hemorrhage complicating intravitreal tissue plasminogen activator and pneumatic displacement of submacular hemorrhage. Retina. 2011;31:2071-7. https:// doi.org/10.1097/IAE.0b013e31822528c8.

17. Fujikawa M, Sawada O, Miyake T, Kakinoki M, Sawada T, Kawamura $\mathrm{H}$, et al. Comparison of pneumatic displacement for submacular hemorrhages with gas alone and gas plus tissue plasminogen activator. Retina. 2013;33:1908-14. https://doi.org/ 10.1097/IAE.0b013e318287d99d.

18. Bennett SR, Folk JC, Blodi CF, Klugman M. Factors prognostic of visual outcome in patients with subretinal hemorrhage. Am J Ophthalmol. 1990;109:33-37.

19. Wu TT, Sheu SJ. Intravitreal tissue plasminogen activator and pneumatic displacement of submacular hemorrhage secondary to retinal artery macroaneurysm. J Ocul Pharmacol Therapeutics. 2005;21:62-67. https://doi.org/10.1089/jop.2005.21.62. 
20. Cho HJ, Koh KM, Kim HS, Lee TG, Kim CG, Kim JW. Antivascular endothelial growth factor monotherapy in the treatment of submacular hemorrhage secondary to polypoidal choroidal vasculopathy. Am J Ophthalmol. 2013;156:524-31. e521. https:// doi.org/10.1016/j.ajo.2013.04.029.

21. Guthoff R, Guthoff T, Meigen T, Goebel W. Intravitreous injection of bevacizumab, tissue plasminogen activator, and gas in the treatment of submacular hemorrhage in age-related macular degeneration. Retina. 2011;31:36-40. https://doi.org/10.1097/ IAE.0b013e3181e37884

22. Kung YH, Wu TT, Hong MC, Sheu SJ. Intravitreal tissue plasminogen activator and pneumatic displacement of submacular hemorrhage. J Ocul Pharmacol Therapeutics. 2010;26:469-74. https://doi.org/10.1089/jop.2010.0066.

23. Peyman GA, Nelson NC Jr, Alturki W, Blinder KJ, Paris CL, Desai UR, et al. Tissue plasminogen activating factor assisted removal of subretinal hemorrhage. Ophthalmic Surg. 1991;22:575-82.

24. Gopalakrishan M, Giridhar A, Bhat S, Saikumar SJ, Elias A,NS. Pneumatic displacement of submacular hemorrhage: safety, efficacy, and patient selection. Retina. 2007;27:329-34. https://doi. org/10.1097/01.iae.0000231544.43093.40.
25. Krepler K, Kruger A, Tittl M, Stur M, Wedrich A. Intravitreal injection of tissue plasminogen activator and gas in subretinal hemorrhage caused by age-related macular degeneration. Retina. 2000;20:251-6.

26. Cho HJ, Koh KM, Kim JH, Kim HS, Han JI, Lew YJ, et al. Intravitreal ranibizumab injections with and without pneumatic displacement for treating submacular hemorrhage secondary to neovascular age-related macular degeneration. Retina. 2015;35:205-12. https://doi.org/10.1097/IAE.0000000000000295.

27. Sacu S, Stifter E, Vecsei-Marlovits PV, Michels S, Schutze C, Prunte C, et al. Management of extensive subfoveal haemorrhage secondary to neovascular age-related macular degeneration. Eye. 2009;23:1404-10. https://doi.org/10.1038/eye.2008.267.

28. Shin JY, Lee JM, Byeon SH. Anti-vascular endothelial growth factor with or without pneumatic displacement for submacular hemorrhage. Am J Ophthalmol. 2015;159:904-14. e901. https:// doi.org/10.1016/j.ajo.2015.01.024.

29. Kim JH, Chang YS, Kim JW, Kim CG, Yoo SJ, Cho HJ. Intravitreal anti-vascular endothelial growth factor for submacular hemorrhage from choroidal neovascularization. Ophthalmology. 2014;121:926-35. https://doi.org/10.1016/j.ophtha.2013.11.004. 